\title{
Los prodigios del hombre encarnado. Víctor Melcior y la redefinición de la mediumnidad (1901)
}

\section{Andrea Graus (*)}

(*) orcid.org/0000-0002-9513-0048. Centre d'Història de la Ciència, Universitat Autònoma de Barcelona. andrea.graus@uab.cat

Dynamis

[0211-9536] 2015; 35 (1): 83-105

http://dx.doi.org/10.4321/S0211-95362015000100004
Fecha de recepción: 16 de septiembre de 2013

Fecha de aceptación: 4 de abril de 2014

SUMARIO: 1.- Introducción. 2.-La dinamita anulada por una potencia invisible. 3.-Los prodigios del hombre encarnado. 4.- Las fuerzas del médium. 5.-Las enfermedades del médium. 6.-La polémica con el «Dr. Bálsamo». 7.-Esa quisicosa llamada el inconsciente del médium. 8.-Conclusiones.

RESUMEN: A finales del siglo XIX, mediante el estudio científico de los fenómenos espiritistas, nuevos enfoques médicos y psicológicos se aplicaron a la mediumnidad. La idea del médium espiritista fue sustituida por la noción del médium como un ser desequilibrado, capaz de emanar fuerzas psíquicas inconscientemente. Este trabajo analiza la redefinición de la mediumnidad a través de unos polémicos artículos del médico catalán Víctor Melcior. Esta microhistoria sirve, por un lado, para situar el debate local dentro del contexto científico internacional y, así, mostrar las relaciones entre el espiritismo, la medicina y la psicopatología del momento. Por otro lado, permite analizar las reacciones de algunos espiritistas a las teorías de Melcior, así como las consecuencias que este debate tuvo para el espiritismo en general.

PALABRAS CLAVE: médium, espiritismo, fuerza psíquica, microhistoria, inconsciente.

KEY WORDS: medium, spiritism, psychic force, microhistory, unconscious. 


\section{Introducción $(*)$}

Aunque la célebre espiritista Amalia Domingo Soler (1835-1909) se refiere a Víctor Melcior i Farré (1860-1929) como «médico espiritista» ${ }^{1}$, algunos de sus planteamientos lo alejaron de dicho movimiento. Este médico nacido en Almenar (Lérida) y afincado en Barcelona, entró en contacto con el espiritismo de la ciudad condal alrededor de 1890. Desde sus primeras experiencias en casa del médico militar y espiritista fervoroso Francesc Parés Llansó (m. 1908), Melcior quedó fascinado por los fenómenos espiritistas, a los cuales por entonces no supo dar una explicación científica. A partir de ese momento, emprendió de forma febril el estudio de cuanta obra de magnetismo, hipnotismo, ciencias ocultas o psicología cayera en sus manos. Tras años de vinculación al espiritismo, desarrolló una aproximación más personal respecto a uno de los puntos clave de la doctrina: la mediumnidad. Como mostraré en este artículo, la definición que hizo del médium lo alejó de sus correligionarios para acercarlo a autores extranjeros que, desde las investigaciones psíquicas, la metapsíquica o la psicopatología francesa abordaron el tema de la mediumnidad mediante nuevos enfoques médicos y psicológicos.

A lo largo de su carrera, Melcior recibió varios premios y distinciones ${ }^{2}$. Aun siendo una figura destacada en el ámbito médico y psiquiátrico de su tiempo, la historiografía de la medicina española parece haberlo olvidado. Respecto a la historiografía del espiritismo, ya de por sí escasa en España, solo Horta ha destacado la vinculación de Melcior con el movimiento espiritista catalán, sin tratarlo en detalle. En relación a la metapsíquica, a la cual Melcior terminó vinculado, Mülberger y Balltondre han expuesto los

$\left(^{*}\right)$ Trabajo realizado en el marco del proyecto subvencionado por el Ministerio de Economía y Competitividad (HAR2009-11342/HIST).

1. Domingo Soler, Amalia. Un héroe ignorado. Luz y Unión. 1901; 2 (35): 60-62 (61).

2. Por ejemplo, en 1885 , tras luchar contra el cólera en Manresa (Cataluña), el ayuntamiento le otorgó un diploma de honor y la junta de auxilios una medalla de plata. En 1889, la Real Academia de Medicina de Barcelona, de la cual fue miembro desde 1900, le premió por su trabajo «Topografía médica de Manresa». En 1907, su memoria titulada: «¿Puede considerarse la voluntad como una fuerza medicatriz?» recibió el ansiado premio de la Real Academia de Medicina de Madrid, el cual además de tener una importante recompensa económica dotaba al premiado con una reconocida reputación dentro de la clase médica. 
debates científicos que esta generó, destacando a sus aliados y detractores dentro de la medicina y la psicología españolas, pero sin mencionarle ${ }^{3}$.

Según el fundador de la doctrina espiritista, Allan Kardec - pseudónimo de Hippolyte Léon Denizard Rivail (1804-1869) -, el médium era un canal para la comunicación espiritual: los espíritus sentían, veían, obraban y hablaban a través de él. La mediumnidad era la capacidad para establecer esta relación. Solía manifestarse de forma espontánea y todas las personas la poseían en mayor o menor medida ${ }^{4}$. Alrededor de 1880, en Estados Unidos e Inglaterra, se fundaron escuelas para su desarrollo. En España, aunque no existió una educación reglada similar, los grandes centros espiritistas ofrecieron talleres semanales dedicados a este fin ${ }^{5}$.

Médiums contemporáneos a Melcior, como Miquel Vives (1842-1906), se convirtieron en ávidos defensores y propagandistas de la mediumnidad espiritista. En sus libros y artículos, se refleja la concepción kardecista del médium como mero intermediario entre el mundo terrestre y el espiritual ${ }^{6}$. Sin duda, la mediumnidad era el punto central del espiritismo, por lo que redefinirla mediante nuevas concepciones procedentes de la medicina y la psicología significó alterar el eje principal de su doctrina. Como mostraré en este artículo, algunos espiritistas no dudaron en atacar a Melcior y a las corrientes médico-psicológicas que sostenían que el médium era el agente productor de los fenómenos espiritistas, en vez del facilitador.

Para la antropología, el trance bajo sus diversas formas de posesión ha sido un objeto central de sus estudios sobre el espiritismo. En especial, la obra de Horta sobre el espiritismo catalán ha sentado las bases para el estudio

3. Por ejemplo, en una de las obras más destacadas sobre hipnotismo y magnetismo en España no se menciona a Víctor Melcior en ningún momento: Montiel, Luis; González de Pablo, Ángel. En ningún lugar, en parte alguna: Estudios sobre la historia del magnetismo animal y del hipnotismo. Madrid: Frenia; 2003. Sobre el espiritismo y la metapsíquica véase: Horta, Gerard. De la mística a les barricades. Barcelona: Proa; 2001, y Cos i revolució. L'espiritisme català o les paradoxes de la modernitat. Barcelona: Edicions de 1984; 2004. Mülberger, Annette; Balltondre Mònica. En el umbral de lo desconocido. Un caso de visión extraordinaria en la España de Primo de Rivera. Dynamis. 2013; 33 (1): 195-216, y Metapsychics in Spain: Acknowledging or questioning the marvellous? History of the Human Sciences. 2012; 25 (2): 108-130.

4. Kardec, Allan. El Libro de los médiums. Málaga: Hojas de Luz; 2009 [edición original de 1861].

5. Por ejemplo, en 1900, el histórico Centro Barcelonés de Estudios Psicológicos dedicaba las sesiones de los jueves «al estudio y desarrollo de mediumnidades». Anónimo. Centro Barcelonés de Estudios Psicológicos. Luz y Unión. 1900; 1 (11): 109.

6. Vives, Miquel. Guía práctica del espiritista. Barberà del Vallés: Humanitas; 1998. 
de la mediumnidad espiritista dentro de este campo ${ }^{7}$. Por lo que respecta a la historia de la ciencia, de la que parte este artículo, los estudios sobre el espiritismo en Europa y Estados Unidos han demostrado que el examen médico y psicológico de los médiums fue una herramienta importante en la generación de conocimiento científico ${ }^{8}$.

Según Monroe, a partir de 1880 el espiritismo kardecista se dividió en dos corrientes: la de carácter moral y la de carácter científico. Esta división se vio impulsada por el interés que mostraron importantes hombres de ciencia, como el químico William Crookes (1932-1919), por los fenómenos del espiritismo. En el contexto anglosajón, la institucionalización científica de estos intereses culminó con la fundación, en 1882, de la Society for Psychical Research. En Francia, la corriente que priorizó el carácter moral del espiritismo estuvo representada por el filósofo espiritista Léon Denis (1846-1927), mientras que el ingeniero Gabriel Delanne (1857-1926) lideró el movimiento que enfatizó su aspecto científico y promovió una aproximación positivista a la doctrina. Desde este punto de vista, las manifestaciones físicas, como las materializaciones o los aportes, cobraron importancia como pruebas empíricas del mundo espiritual ${ }^{9}$.

Dentro del llamado «espiritismo científico», además de priorizar una aproximación empírica a la doctrina, se destacó su carácter psicológico. La historiografía ha mostrado cómo el desarrollo de las teorías sobre el automatismo, la autosugestión o el inconsciente estuvo fuertemente influenciado por los fenómenos espiritistas ${ }^{10}$. Aunque desde puntos de vista contrarios, autores como Frederic W. H. Myers (1943-1901) o Pierre Janet (1859-1947) abordaron los aspectos psicológicos del espiritismo partiendo de sus particulares concepciones del inconsciente ${ }^{11}$. Mediante esta clase de

7. Horta, n. 3

8. Véase, por ejemplo: Plas, Régine. Naissance d'une science humaine: la Psychologie. Les psychologues et le 'merveilleux psychique'. Rennes: Presses Universitaires de Rennes; 2000. Owen, Alex. The place of enchantment. London: University of Chicago Press; 2004. Wolffram, Heather. The stepchildren of science. Amsterdam, New York: Rodopi; 2009.

9. Monroe, John Warne. Laboratories of faith. Mesmerism, spiritism, and occultism in modern France. Ithaca and London: Cornell University Press; 2008, p. 221-226. Beloff, John. Parapsychology. A concise history. London: The Athlone Press; 1993, p. 226-233.

10. Ellenberger, Henri F. El descubrimiento del inconsciente. Madrid: Gredos; 1976.

11. Bacopoulos-Viau, Alexandra. Automatism, surrealism and the making of french psychopathology: The case of Pierre Janet. History of Psychiatry. 2012; 23 (3): 257-276. Shamdasani, Sonu. Automatic writing and the discovery of the unconscious. Spring. 1993; 54 (2): 100-131. 
planteamientos el énfasis se puso en el psiquismo de médium. Este principio es la base sobre la cual Melcior asentó su particular visión del espiritismo.

Si bien Janet admitió la dificultad de intentar conciliar la psicología fisiológica y experimental con la investigación psíquica de los fenómenos espiritisas $^{12}$, Melcior nunca abandonó esta ambición. En este artículo me propongo presentar a este médico como a uno de los autores españoles más originales y críticos respecto al estudio científico del espiritismo. Lo haré a través de una microhistoria que refleja la polémica que generaron una serie de artículos de Melcior, titulados «La dinamita anulada por una potencia invisible» (1901), publicados en la revista espiritista Luz y Unión. En ellos, defendió que el causante de los fenómenos espiritistas era el médium, al cual definió como un ser desequilibrado capaz de emanar fuerzas desconocidas de carácter psíquico. Hay que resaltar que la idea de la mediumnidad presente en estos artículos se vio reflejada en sus posteriores publicaciones sobre el tema; especialmente, su mayor obra al respecto: Los estados subconscientes y las aberraciones de la personalidad (1904) ${ }^{13}$.

Aun el carácter microhistórico de este estudio, el caso permite explorar, de modo inductivo, los procesos macrohistóricos imbricados ${ }^{14}$. En la línea de los enfoques en historia de la ciencia citados, muestro la relación entre la medicina, la psicología y la mediumnidad a través de los artículos de Melcior y sus influencias. Asimismo, analizo las polémicas que generaron dichos artículos entre algunos espiritistas y sus consecuencias para la doctrina espírita.

\section{La dinamita anulada por una potencia invisible}

Como he mencionado, en 1901, Melcior publicó en Luz y Unión una serie de artículos titulados: «La dinamita anulada por una potencia invisible».

12. Monroe, n. 9, p. 211.

13. Para indagar más en esta cuestión véase: Graus, Andrea. ¿Dobles o espíritus? Las teorías del desdoblamiento frente al espiritismo en la España de principios del siglo XX. Asclepio. 2014; 66 (1): 1-35.

14. Sobre la historiografía y la microhistoria véase, por ejemplo: Gray, Marion W. Microhistory as universal history. Central European History. 2001; 34 (3): 419-431. Kusch, Martin. Reflexivity, relativism, microhistory: Three desiderata for historical epistemologies. Erkenn. 2011; (75): 483494. Lepore, Jill. Historians who love too much: Reflections on microhistory and biography. The Journal of American History. 2001; 88 (1): 129-144. 
Dichos artículos partían de un fenómeno observado, los días 10 y 17 de mayo de 1893, bajo la presencia del «médium J.». El fenómeno consistió en la anulación de la explosión de dos cargas, la primera de pólvora y la segunda de dinamita, que Melcior y otros asistentes habían colocado en las ruinas del castillo de Montcada.

Cabe suponer que, al hablar del «médium J.», Melcior se refería a Juanito Grau, con quien había compartido sesiones desde 1890. Estas experiencias se llevaron a cabo en el domicilio del médico militar Francesc Parés Llansó. Algunos de los participantes de las sesiones fueron el célebre espiritista Vizconde de Torres-Solanot y el matrimonio Grau. Actuaron como médiums la esposa de Parés y Juanito, hijo menor de los Grau.

La mediumnidad de Juanito Grau no era la más valorada por el espiritismo kardecista, ya que era de fenómenos físicos. Según Kardec, el principal propósito de las sesiones era el de obtener lecciones morales a través de los mensajes de los espíritus. A su parecer, el estatus de los médiums de fenómenos físicos era inferior al de los parlantes o escribientes, pues sus formas de comunicación - por ejemplo, mediante golpes en muebles o paredes llamados raps - eran menos efectivas que la escritura o el habla. Este aspecto del espiritismo kardecista contrasta con la corriente espiritista dominante en el mundo anglosajón, el «spiritualism», donde los médiums de fenómenos físicos, como Daniel Dunglas Home (1833-1886) tuvieron un papel muy destacado y en ningún caso inferior ${ }^{15}$.

En el momento en que Melcior entró en contacto con el espiritismo, una estrategia de promoción del movimiento era hacer propaganda de su doctrina a través de la moral kardecista, basada en la creencia en el progreso y el perfeccionamiento del hombre a través de la reencarnación; como puede observarse en las actas del Primer Congreso Espiritista Internacional (Barcelona, 1888), el cual resultó un gran acto de propaganda espírita ${ }^{16}$. A diferencia de lo promulgado por Kardec, Melcior siempre prefirió estudiar a los médiums de fenómenos físicos que no a los parlantes o escribientes. El motivo es que, a su parecer, estos les permitían llevar a cabo un estudio imparcial del espiritismo, ya que sus fenómenos eran más objetivables y, a priori, estaban vacíos de contenido. Su participación en el Grupo Barcelonés de Investigaciones Psíquicas, vinculado a la entidad espiritista Centro

15. Véase, por ejemplo: Owen, n. 8

16. Primer Congreso. Primer Congreso Internacional Espiritista: representaciones, adhesiones, sesiones públicas. Barcelona: Imprenta de Daniel Cortezo y Cia; 1888. 
Barcelonés de Estudios Psicológicos, nos muestra su preferencia por el punto de vista adoptado por las sociedades dedicadas al estudio científico de los fenómenos, como la Society for Psychical Research ${ }^{17}$.

Según relata Melcior en sus artículos, el 10 de mayo de 1893 el grupo que se reunía en casa de Parés recibió una comunicación del espíritu guía ${ }^{18}$ a través del «médium J.» - suponemos, Juanito Grau-. Este les anunció que deseaba darles «una prueba evidente del poder que dimana del mundo espiritual, a cuyo efecto, anularía la fuerza expansiva de la pólvora» ${ }^{19}$. Atraído por el desafío, el grupo se dirigió a las ruinas del castillo de Montcada, cerca de Barcelona. Nada más llegar, construyeron dos barrenos, en los cuales colocaron dos cargas de pólvora. Tras detonar la primera carga vieron, incrédulos, cómo el barreno permaneció intacto, sin que se hubiera producido la explosión. Según les comunicó el espíritu guía, para comprobar que la pólvora no había sido trucada no anularía la explosión de la segunda carga. Al detonarla, la pólvora estalló y se formaron varias grietas alrededor del barreno, el cual quedó destrozado.

Comprobada la supuesta acción espiritual sobre la pólvora, Melcior preguntó al espíritu guía si sería capaz de anular la potencia de la dinamita, a lo cual este respondió afirmativamente. El 17 de mayo, el grupo de estudio construyó un nuevo barreno en la misma localización. Colocaron una carga de ciento veinte gramos de dinamita la cual, como en el caso de la primera carga de pólvora, tampoco estalló. Tras la experiencia, Melcior quedó convencido de que no se trataba de un fraude; sin embargo, dudó de que el fenómeno estuviera relacionado con la intervención de los espíritus ${ }^{20}$.

\section{Los prodigios del hombre encarnado}

$\mathrm{Al}$ intentar dar una explicación al fenómeno de la dinamita, Melcior lo planteó de la siguiente manera: «De momento tenemos como factor la existencia

17. Anónimo. Grupo Barcelonés de Investigaciones Psíquicas. Revista de Estudios Psicológicos. 1895; (29), p. 111-114.

18. El espíritu guía era considerado el espíritu intermediario entre el médium y los espíritus.

19. Nota al pie de Melcior añadida por él en su traducción a la obra de Rochas: Rochas, Albert de. La exteriorización de la motilidad. Barcelona: Imprenta de Pujol y Cia; 1897 [edición original de 1896], p. 254.

20. Melcior i Farré, Víctor. La dinamita anulada por una potencia invisible. Luz y Unión. 1901; 2 (46): 318-320. 
de una fuerza. Esta fuerza debió ser desarrollada, bien por inteligencias del espacio, o por el espíritu de los asistentes» ${ }^{21}$. Estas dos opciones correspondían a las dos grandes corrientes que existían, a finales del siglo XIX, para explicar el origen de los fenómenos mediúmnicos ${ }^{22}$. La primera opción se relacionaba con la hipótesis espírita, por la cual los espíritus serían los causantes de los fenómenos. La segunda representaba un conglomerado de las distintas teorías que postulaban que estos fenómenos serían debidos a la emanación de una fuerza natural, aún desconocida, por parte de las personas en general y en mayor intensidad por parte de los médiums ${ }^{23}$. Melcior optó por esta última explicación, la cual se expondrá en el siguiente apartado. A diferencia de algunos de sus contemporáneos, como el fisiólogo Charles Richet (1850-1935), no negó del todo la hipótesis espiritista y se declaró partidario de algunos aspectos de su doctrina, como la reencarnación ${ }^{24}$.

$\mathrm{Al}$ analizar la hipótesis espiritista, en relación al fenómeno de la dinamita, Melcior postuló que, una vez desencarnado, el espíritu era capaz de producir manifestaciones inteligentes, como las comunicaciones propias de las sesiones espiritistas. Por lo tanto, afirmó que los espíritus habrían podido causar el fenómeno de la dinamita. Sin embargo, en sus artículos quiso ir un paso más allá. Comparando la capacidad de actuación de los encarnados (las personas) con la de los desencarnados (los espíritus), preguntó: «Ahora bien: ¿los encarnados no pueden obrar de igual manera?» ${ }^{25}$. Con ello, planteó la posibilidad de que una persona fuera capaz de producir los prodigios atribuidos a los espíritus.

Según Melcior, había un aspecto que tanto los encarnados como los desencarnados compartían: la voluntad. El hecho de que daba especial importancia a este tema puede comprobarse en su memoria titulada: ¿Puede la voluntad actuar como fuerza medicatriz? (1908), donde expuso las potencias de la voluntad como herramienta terapéutica. En ella la definió

\footnotetext{
21. Melcior, n. 20, p. 320.

22. Wolffram, Heather. Hallucination or materialization? The animism vs. spiritism debate in late19th-century Germany. History of the Human Sciences. 2012; 25 (2): 45-66.

23. Alvarado, Carlos. Human radiations: Concepts of force in mesmerism, spiritualism and psychical research. Journal of the Society for Psychical Research. 2006; 70.3 (884): 138-162.

24. Los espiritistas creían en un tipo de reencarnación basada en el progreso y la pluralidad de mundos habitados en el universo. Durante la reencarnación, la conciencia de la persona no se perdía. De este modo, uno seguía siendo el mismo aunque no tuviera ningún recuerdo de sus vidas anteriores. Sharp, Lynn. Secular spirituality: Reincarnation and spiritism in nineteenth-century France. Lanham, MD: Lexington Books; 2006.

25. Melcior, n. 20, p. 320.
} 
como «un facultad anímica que se resuelve en voliciones, cada una de las cuales supone un estado fisiológico adecuado de los centros nerviosos» ${ }^{26}$. Como se verá más adelante, esta imbricación de conceptos psicológicos y fisiológicos es la misma que aplicó al tema de la mediumnidad.

La mencionada memoria fue premiada por la Real Academia de Medicina en 1907. Aun así, fue duramente criticada. En la introducción, el médico Manuel Iglesias y Díaz (1830-1922) cargó contra la aproximación ante todo teórica de Melcior, faltada de ejemplos experimentales. En palabras de Iglesias, el texto adolecía de «psicologismo» ${ }^{27}$, en cuanto lo que más le interesó resaltar era el aspecto psicológico del tema. A su parecer, Melcior no había tenido en cuenta que su trabajo iba dirigido a profesionales de la medicina, de modo que se había embarcado en «teorías y especulaciones metafísicas, las cuales son siempre propensas a ese conceptualismo personal, que tanto ha servido para obscurecer las doctrinas médicas a través de los siglos» ${ }^{28}$.

La introducción de Iglesias nos muestra que Melcior fue una figura incómoda para buena parte de la comunidad médica, en especial para la psiquiátrica, por la cual tanto se interesó Melcior a través de su amigo Abdón Sánchez Herrero (1852-1904) ${ }^{29}$. Como ha mostrado Huertas, a finales del siglo XIX la psiquiatría española priorizó un enfoque práctico y positivista ${ }^{30}$. Teniendo en cuenta este aspecto, resulta comprensible que Iglesias tachara la memoria de Melcior de oscurantista.

26. Melcior i Farré, Víctor. ¿Puede considerarse la voluntad como una fuerza medicatriz?: Terapéutica de esa potencia anímica, cuando está debilitada y perturbada, introducción de Manuel Iglesias, Madrid: Enrique Teodoro; 1908, p. 120. Manuel Iglesias fue miembro de la Real Academia de Medicina desde 1873, en la cual ejerció cargos de bibliotecario y secretario. Su labor como médico y cirujano se desarrolló, principalmente, en el Hospital de la Princesa de Madrid.

27. Introducción de Iglesias en el libro de Melcior: Melcior, n. 26, p. 3.

28. Introducción de Iglesias en el libro de Melcior: Melcior, n. 26, p. 3.

29. Sánchez Herrero fue un catedrático de Clínica Médica en la Universidad Valladolid. En 1888 publicó El hipnotismo y la sugestión. Influenciado por la Escuela de Nancy, se conviritió en uno de los principales referentes de la hipnoterapia en España. Véase: Graus, Andrea. Hypnosis in Spain (1888-1905). From spectacle to medical treatment of mediumship. Studies in History and Philosophy of Science Part C: Studies in History and Philosophy of Biological and Biomedical Sciences. 2014; 48: 85-93. Montiel; González de Pablo, n. 3. Con Melcior compartió su interés por el uso terapéutico de la hipnosis y el estudio de los fenómenos extraordinarios asociados al hipnotismo, como la clarividencia. A este respecto, puede consultarse el prólogo que Sánchez Herrero hizo a la traducción anotada de Melcior del libro de Rochas, n. 19.

30. Huertas García-Alejo, Rafael. La psiquiatría española en al siglo XIX. Primeros intentos de institucionalización. En: Sociedad de Historia y Filosofía de la Psiquiatría. Congreso. Un siglo de psiquiatría en España. Madrid: Extra Editorial; 1995, p. 21-47. 
Como se ha mencionado, para Melcior era importante «admitir que el hombre encarnado es capaz de realizar verdaderos prodigios» ${ }^{31}$, porque «si creemos que nos hallamos siempre en presencia de espíritus del espacio, acabamos por renunciar a nuestra voluntad» ${ }^{32}$. Para ejemplificar su concepción de que un encarnado era capaz de producir los prodigios que se le atribuían a los espíritus, en sus artículos expuso varias experiencias de los que consideraba los maestros del «magnetismo transcendental», entre ellos: el Barón Jules du Potet (1796-1881), William Crookes (1832-1919), Hector Durville (1849-1923) y Albert de Rochas d'Aiglun (1837-1914) ${ }^{33}$. Desde perspectivas a veces distintas, los autores mencionados contribuyeron a generar la idea de que las personas poseían potencias o fuerzas latentes, capaces de actuar sobre la materia y producir los fenómenos asociados al espiritismo. En el siguiente apartado indago en esta cuestión. Fueron las experiencias de dichos autores, así como las propias investigaciones de Melcior, las que lo llevaron a afirmar: «No busquemos [el origen del fenómeno espiritista] en lo exterior, ni en lo superior, ni en lo inferior; busquémoslo en lo interior del ser, llamado médium, porque él es, y no otro, quien produce a sabiendas o sin saberlo, el fenómeno que queda narrado» ${ }^{34}$.

Por tanto, hay que matizar el posicionamiento de Melcior: los fenómenos propios del espiritismo serían producto de un encarnado, pero no de uno cualquiera, sino de un médium. Como veremos, la manera en que Melcior concibió a los médiums le generó más de un conflicto con los espiritistas más doctrinarios.

\section{Las fuerzas del médium}

La siguiente cita resulta útil para ver cómo Melcior definió la mediumnidad:

31. Melcior, n. 20, p. 320.

32. Melcior i Farré, Víctor. La dinamita anulada por una potencia invisible. Luz y Unión. 1901; 2 (51): 445-446, p. 446

33. Melcior i Farré, Víctor. La dinamita anulada por una potencia invisible. Luz y Unión. 1901; 2 (47): 343-346. Melcior i Farré, Víctor. La dinamita anulada por una potencia invisible. Luz y Unión; 1901, 2 (48): 369-372.

34. Melcior i Farré, Víctor. La dinamita anulada por una potencia invisible. Luz y Unión. 1901; 2 (50), 414-416, p. 414-415. 
«Los médiums son seres que producen corrientes de una especie de electricidad que no ha quedado definida aún. Esas corrientes deben fraguarse en el sistema bulbo medular, e induce a suponer que esto sea así la circunstancia de que en todos los médiums de efectos físicos existe el predominio de funciones de los órganos de la vida vegetativa; el antecedente o el presente de una afección nerviosa, profunda, tal como neurastenia, histeria o histeroepilepsia, y de rebote se observa una obnubilación, suspensión o retardo en las funciones de los altos centros cerebrales (reflexión y voluntad)» ${ }^{35}$.

Cuatro son los elementos característicos de esta definición que pueden hallarse en las definiciones de científicos contemporáneos a Melcior interesados por la mediumnidad. Primero, que los fenómenos son producidos mediante la emanación de una fuerza desconocida, parecida a la electricidad, por parte del médium; segundo, que esta fuerza se genera en el bulbo raquídeo; tercero, que el médium sufre una dolencia psicológica semejante a la histeria; cuarto, que la producción de los fenómenos requiere de la anulación momentánea de las funciones superiores del médium, en otras palabras, que requiere del trance. Dada su importancia, analizo el segundo y el tercer punto por separado en el siguiente apartado. En este, expongo el primer y el cuarto punto en relación a un contexto más amplio.

Como ha mostrado Alvarado, la idea de que del médium irradiaba una fuerza semejante a la electricidad estaba muy presente en los círculos científicos de finales del siglo XIX ${ }^{36}$. En España, uno de los defensores de esta clase de teorías fue Josep Comas i Solà (1868-1937), astrónomo catalán muy polémico con los espiritistas ${ }^{37}$. Según Comas, este tipo de explicaciones fueron las más aceptadas entre la comunidad científica internacional. En sus palabras: «Llámese a esta fuerza: fuerza psíquica [de Crookes], fuerza ódica [de Reichenbach], fuerza néurica [de Baréty], lo cierto es que en el fondo hay concordancia en admitir una emanación por parte del médium» ${ }^{38}$.

En el caso de Melcior, su mayor influencia en este aspecto fue Albert de Rochas. Formado como ingeniero militar, Rochas fue conocido por sus trabajos en el campo de las investigaciones psíquicas. De entre sus obras, la

\footnotetext{
35. Melcior, n. 34, p. 415.

36. Alvarado, n. 23, p. 138-162.

37. Roca, Antoni. Los ecos de un debate internacional. En: Comas i Solà, Josep, El espiritismo ante la ciencia. Barcelona: Altafulla; 1986, p. V-XXVII.

38. Comas i Solà, Josep. El espiritismo ante la ciencia: Estudio sobre la mediumnidad. Barcelona: F. Granada; 1908, p. 316.
} 
que más marcó a Melcior fue L'extériorisation de la motricité (1896). En ella, Rochas narró sus experiencias con diversos sujetos hipnotizados, así como las sesiones con médiums como Eusapia Palladino, en las cuales participaron personalidades científicas como Richet o Lombroso. Sus investigaciones estuvieron influidas por la teoría sobre la fuerza la fuerza psíquica de William Crookes. Dicha fuerza, natural aunque desconocida, estaría ligada al organismo humano. Todas las personas la poseerían en distinto grado, manifestándose en mayor intensidad en los individuos llamados médiums. En su grado máximo, provocaría los fenómenos asociados al espiritismo ${ }^{39}$.

Según Melcior, los diferentes grados de emanación de la fuerza psíquica permitían categorizar los sujetos en tres «tipos psíquicos»: el débil, el medio y el fuerte. En el primer caso, la fuerza predominante sería centrífuga en vez de centrípeta, lo cual favorecería su exteriorización. Al igual que Rochas, Melcior defendió que la inestabilidad en el carácter y la debilidad de la voluntad facilitaban este proceso. Así, no es de sorprender que colocase a los enfermos mentales en este primer grupo. El tipo medio, en cambio, representaría la estabilidad mental y las personas equilibradas. Por último, el tipo fuerte correspondería a aquellos individuos de mente cerrada en los que predominaría la fuerza centrípeta sobre la centrífuga, hecho que dificultaría su irradiación ${ }^{40}$. Los diferentes grados de la emanación de la fuerza producirían distintos fenómenos propios del espiritismo. Las manifestaciones más rudimentarias serían raps, mientras que, en su grado máximo, se llegaría a exteriorizar completamente el yo en forma de «doble fluídico»o «fantasma de un vivo» ${ }^{41}$.

Para probar que el médium era el causante de los fenómenos espiritistas Melcior ideó una sencilla experiencia, la cual podía llevarse a cabo en cualquier casa que se creyera embrujada. Por aquel entonces, los fenómenos de las casas encantadas no solo interesaban a los espiritistas, sino que la propia Society for Psychical Research había creado un comité científico dedicado a su estudio ${ }^{42}$. En su misma línea, Melcior creyó que los fenómenos de las casas embrujadas eran debidos a «la presencia de una o más personas

39. Crookes, William. Researches in the phenomena of spiritualism. London: The Quarterly Journal of Science; 1874.

40. Melcior i Farré, Víctor. La enfermedad de los místicos (Patología-psíquica). Barcelona: Establecimiento Tipográfico de Juan Torrents y Coral; 1900, p. 239-240.

41. Sobre este tema véase: Graus, n. 13.

42. Beloff, n. 9, p. 72. 
que proyectan sus fluidos en aquella mansión» ${ }^{43}$. Para probar esta teoría propuso desalojar las casas de las personas que las habitaban y comprobar si, en presencia de otras, se producía algún fenómeno. Según Melcior, esta sencilla experiencia evidenciaría que:

«En el plano terráqueo, un encarnado puede poder más que un desencarnado, y no hay en esto exageración alguna. Basta recordar que para toda manifestación del mundo espiritual se requiere indispensablemente la presencia de un médium, y nunca jamás ha tenido lugar un fenómeno espontáneo, sin la correspondiente ayuda de la energía psíquica del médium» ${ }^{44}$.

La emanación de la energía o fuerza psíquica requería, según Melcior, de la suspensión momentánea de las facultades superiores del médium, es decir, requería del trance - cuarto punto antes señalado.

El estado de trance había sido bautizado como hémisomnambulisme por Richet, noción también defendida por Janet ${ }^{45}$. En este estado, el médium daba la impresión de estar consciente y era capaz de desarrollar acciones complejas de forma involuntaria, como la escritura automática. Como Richet, Melcior creía que ningún médium podía dar lugar a manifestaciones inteligentes sin que se produjera el estado de trance donde sus facultades superiores quedaban suspendidas ${ }^{46}$. En sus palabras: «esa misma fuerza, (electricidad, magnetismo o lo que fuere) se desprende de un modo vigoroso en algunos organismos desequilibrados que llevan la característica de tener suspendidas o amortiguadas las funciones más elevadas del sensorio» ${ }^{47}$. En este sentido, es importante destacar que la emanación de la fuerza se hacía de forma inconsciente, un tema en el que se profundizará más adelante. Como se observa en la cita, Melcior no sabía de qué clase de fuerza se trataba, pero tenía claro que solo la irradiaban personas mentalmente inestables, tema que expongo en el siguiente apartado.

\footnotetext{
43. Melcior, n. 34, p. 415.

44. Melcior, n. 34, p. 416, cursiva en el original.

45. Richet, Charles. Traité de metápsychique. Paris: Félix Alcan; 1922, p. 137. Janet, Pierre. L'Automatisme psychologique. Paris: Félix Alcan; 1898 [edición original de 1889], p. 125.

46. La anulación momentánea de las funciones cerebrales superiores del médium también era el principio fundamental del magnetismo animal estudiado por los médicos del romanticismo alemán. Véase: Montiel, Luis. Magnetizadores y sonámbulas en la Alemania Romántica. Madrid: Frenia; 2008.

47. Melcior, n. 34, p. 415.
} 


\section{Las enfermedades del médium}

La asociación que Melcior hizo entre la mediumnidad y las psicopatías - tercer punto antes señalado- es la que le generó más enemistades con los espiritistas. Sobre esta cuestión siempre se mostró tajante. Son varias las ocasiones en que repitió que los fenómenos del espiritismo «no puede nunca producirlos una persona sana, sino que debe producirlos un sujeto enfermo con dolencia psicológica» ${ }^{48}$.

La relación entre la mediumnidad y las psicopatías cobró importancia a finales del siglo XIX en Francia. Desde el campo de la psicología anormal, médicos y psiquiatras como Jean-Martin Charcot (1825-1893), Charles Féré (1852-1907), Alfred Binet (1857-1911) o Pierre Janet defendieron la asociación entre la mediumnidad y la histeria ${ }^{49}$. De entre estos autores, cabe destacar a Janet por ser quien más influenció a Melcior. En L'Automatisme psychologique (1889), Janet estableció la relación entre el desdoblamiento de la personalidad, en sentido psicopatológico, y el espiritismo ${ }^{50}$. Aplicada a la mediumnidad, la teoría del desdoblamiento suponía que, cuando el médium entraba en trance, una personalidad subconsciente lo dominaba ${ }^{51}$. Según Melcior, los espiritistas solían confundir el desdoblamiento con la posesión ya que, durante el trance, los médiums acostumbraban a hablar de ellos mismos en tercera persona. En el contexto español, otros médicos distinguidos defendieron esta posición. Entre ellos destacan Manuel Tolosa Latour (1857-1919) y Jeroni Estrany (1857-1918). Para ambos no había médium que no fuera a su vez un histérico o que, como opinaba Melcior, sufriera alguna dolencia de carácter psicológico ${ }^{52}$.

Los médiums no siempre se rebelaron contra esta clase de teorías. En Italia, tanto el clarividente Pickmann como Eusapia Palladino confesaron padecer alguna clase de psicopatía, aunque no admitieron que esta fuera la

\footnotetext{
48. Melcior, n. 34, p. 415

49. Le Maléfan, Pascal. Folie et spiritisme: Histoire du discours psychopatologique sur la practique du spiritisme, ses abords et ses avatars: 1850-1950. Paris et Montréal: L'Harmattan; 1999.

50. Leblanc, André. The origin of the concept of dissociation: Paul Janet, his nephew Pierre, and the problem of post-hypnotic suggestion. History of Science. 2001; 39: 57-67.

51. Janet, n. 45, p. 122-126.

52. Tolosa Latour, Manuel. Las fuerzas físicas y la fuerza psíquica. Madrid: Gutenberg; 1884. Estrany, Jeroni. Carta abierta. En: Comas, n. 38, p. 12-147.
} 
causante de sus facultades mediúmnicas ${ }^{53}$. Con una actitud más combativa, en Francia los espiritistas desarrollaron sus propias terapias para luchar contra las teorías que pretendían patologizar la mediumnidad. Según Edelman, gracias a este proceso apareció la figura del médico espiritista, encargado de presentar el espiritismo como una cura orgánica y psicológica, además de espiritual. Dándole la vuelta a los argumentos de Janet, espiritistas franceses, como el médico Louis Théodore Chazarain, instaron a los histéricos a aceptar que sus supuestas personalidades subconscientes no eran más que espíritus que intentaban comunicarse con ellos ${ }^{54}$. El desarrollo de la mediumnidad, es decir, el aprender a canalizar los espíritus, se convirtió en la terapia recomendada. Como representante español de esta corriente, cabe destacar la figura del médium Jacint Fornaguera ${ }^{55}$.

Hay que considerar que, si bien Melcior asoció la mediumnidad con las psicopatías, no por ello dejó de ser un miembro apreciado dentro del movimiento espiritista. En 1915, algunos socios del Centro Barcelonés de Estudios Psicológicos propusieron que se le nombrara presidente honorario $^{56}$. No obstante, sus polémicas opiniones suscitaron respuestas. En uno de los artículos en donde defendió la relación entre la mediumnidad y las psicopatías, la redacción de Luz y Unión añadió la siguiente nota al pie: «Dejamos al distinguido colaborador nuestro, autor del presente trabajo, la responsabilidad de la teoría que atribuye a una dolencia psicológica la posesión de facultades medianímicas, cuya apreciación creemos errónea. - (N. de la R.)» ${ }^{57}$.

Un precedente de esta clase de afirmaciones en Melcior puede hallarse en su libro: La enfermedad de los místicos (Patología-psíquica) (1900). En él, abordó el tema del misticismo a través de la fisiología patológica y la psicología anormal. En éste se halla el germen de las explicaciones fisiológicas que Melcior dio a la mediumnidad, como la que postulaba que la fuerza psíquica se formaba en el bulbo raquídeo - segundo punto antes

53. Valentí Camp, Santiago; Massaguer, Enrique. Las sectas y las sociedades secretas a través de la historia. Barcelona: Antonio Virgili; 1912, p. 241-242.

54. Edelman, Nicole. Lo oculto y las terapéuticas espiritistas del espíritu y del cuerpo en Francia (1850-1914): De la creencia al saber y vuelta. Asclepio. 2006; LVIII (2): 39-62; Voyantes, guérisseuses et visionnaires en France 1775-1814. Paris: Albin Michel; 1998.

55. Fornaguera, Jacint. El espiritismo ante la ciencia: Sobre un juicio crítico publicado por D. J. Comas Solá. Barcelona-San Martín: Tip. La Moderna; 1908, p. 28-29.

56. Anónimo. Crónica. La Evolución. 1915; XII (8): 62-63, p. 63.

57. Nota al pie de la Redacción de Luz y Unión en el artículo de Melcior: Melcior, n. 32, p. 445. 
señalado-. Para formular esta teoría, Melcior se basó en la explicación fisiológica que se dio al caso de la mística belga Louise Lateau (1850-1883), quien a la edad de 18 años empezó a padecer una serie de éxtasis y estigmas. Según narra Melcior, los médicos llegaron a la conclusión de que los síntomas eran fruto de una neuropatía que tenía su origen en la excitación del bulbo raquídeo ${ }^{58}$.

Esta misma teoría era aplicable, en su opinión, al trance de los médiums. En una nueva mezcla de conceptos psicológicos y fisiológicos, defendió que, a nivel biológico, el trance podía explicarse mediante la ley de los equivalentes vitales del médico Josep Letamendi (1828-1897). Entendida como un principio general, dicha ley suponía que los seres vivos disponen de un caudal de energía limitado. La energía no puede multiplicarse sino que debe de quedar repartida entre las distintas funciones, de ahí que, si aumenta en una función, automáticamente se resta en otra ${ }^{59}$. Melcior se basó en esta ley para afirmar que: «Tan pronto como a un organismo se le suspenden o amortiguan las mencionadas funciones del intelecto, viene como secuela necesaria la hipertrofia de función en las regiones bulbo-medulares y se entra en el reinado del automatismo» ${ }^{60}$. Es decir: cuando el médium entraba en trance, su energía disminuía en las regiones superiores, hecho que provocaba un aumento de la energía en la región bulbo-medular, la cual causaba la hipertrofia del bulbo raquídeo. Según Melcior: «En tal situación se está propenso a los desprendimientos de la citada energía psíquica, que puede llegar a chorrear continuamente originando fenómenos insólitos» ${ }^{61}$.

A modo de resumen, puede concluirse que para Melcior el médium era un ser psíquica y fisiológicamente enfermo, cuyo desequilibro favorecía la emisión inconsciente de una fuerza, constituida en el bulbo raquídeo, productora de los fenómenos propios del espiritismo. Es evidente que esta forma de entender la mediumnidad estaba muy alejada de la doctrina de Kardec. Como veremos a continuación, hubo algún espiritista que ante los artículos de Melcior no dudó en mostrar su rabia y consternación.

\footnotetext{
58. Melcior, n. 40, p. 30-33.

59. Enríquez de Salamanca, Dr. La castidad ante la ciencia médica. Acción Española. 1934; VIII (48): $1181-1182$.

60. Melcior, n. 34, p. 415.

61. Melcior, n. 34, p. 415, cursiva en el original.
} 


\section{La polémica con el «Dr. Bálsamo»}

Tras la publicación de la serie de artículos titulados «La dinamita anulada por una potencia invisible», la revista Luz y Unión recibió una carta polémica. El firmante era el «Dr. Bálsamo». Supuso Melcior, que el nombre era un pseudónimo. Según afirmó, teniendo en cuenta la calidad de los argumentos esgrimidos era poco probable que se tratara de una persona con el título de doctor ${ }^{62}$. Aun así, Bálsamo firmó otros artículos no dirigidos a Melcior mediante este nombre, por lo que, de tratarse de un pseudónimo, hay que considerar que no fue elegido únicamente para la ocasión.

La carta de Bálsamo, titulada «Desvío lamentable», fue publicada en la portada de Luz y Unión. En ella atacó dos aspectos capitales en los artículos de Melcior: primero, el uso de las teorías del inconsciente para explicar los fenómenos espiritistas; segundo, la asociación, propia de la psicopatología francesa, entre la mediumnidad y las psicopatías.

En el primer caso, expresó su malestar respecto a la tendencia que se había impuesto, entre los hombres de ciencia, a atribuir los fenómenos del espiritismo al inconsciente del médium mediante teorías como la de la fuerza psíquica de Crookes y el desdoblamiento de la personalidad de Janet. Según Melcior, Bálsamo tenía una idea muy difusa del inconsciente, ya que en varias ocasiones lo equiparó al automatismo o a la noción ocultista del cuerpo astral $^{63}$. Para Melcior, este hecho demostraba que Bálsamo desconocía este concepto, al cual llegó a referirse como «esa quisicosa llamada inconsciente [... que] se ha pegado fuertemente a algunos sabios del espiritualismo moderno» ${ }^{64}$. En el siguiente apartado profundizo sobre este tema.

En un segundo plano, Bálsamo criticó la asociación entre la mediumnidad y las dolencias psicológicas. En una parodia constante de las explicaciones fisiológicas apuntadas por Melcior, aseguró haber visto «varios médiums tan equilibrados y tan armónicos en su funcionalismo orgánico que bien lo quisieran para sí muchos animistas a la moderna» ${ }^{65}$. En contraposición a la idea de la mediumnidad como causa de dolencias psicológicas, se sumó

62. Melcior i Farré, Víctor. Cuatro palabras. Luz y Unión. 1901; 2 (53): 491-492 (491). El uso de pseudónimos era una práctica habitual. Algunos ejemplos de espiritistas conocidos serían: Quintín López Gómez (Quilogo), José Cembrano (Nikto), Eduardo Anaya (Profesor Asmara).

63. Melcior, n. 62, p. 492.

64. Bálsamo, Dr. Desvío lamentable. Luz y Unión. 1901; 2 (52): 457-460 (458). Cursiva en el original.

65. Bálsamo, n. 64, p. 459. 
a las corrientes espiritistas francesas que la consideraban como una forma de terapia ${ }^{66}$.

Aunque la mayoría de argumentos esgrimidos por Bálsamo se basaron en ridiculizar los de Melcior, de sus palabras se destilan algunas cuestiones interesantes. Por ejemplo, la asociación entre la modernidad y los supuestos enemigos del espiritismo. En su carta se refirió a los partidarios de las teorías del inconsciente mediante expresiones como: «sabios del espiritualismo moderno»o «animistas a la moderna» ${ }^{67}$. En estos casos el término «moderno» se usó en un sentido peyorativo. Como muestro en el siguiente apartado, este hecho se debió a que Bálsamo creyó que los teóricos del inconsciente, símbolo de la modernidad ${ }^{68}$, llevaron a cabo una apropiación descarada de los conceptos del espiritismo.

La carta de Bálsamo termina con un comentario maliciosamente dirigido hacia Melcior: «No se olvide de que los que presumen de más listos son los que con mayor facilidad se enredan» ${ }^{69}$. La escueta respuesta de este último, titulada: «Cuatro palabras», fue publicada en el siguiente número de Luz y Unión. En ella, afirmó que el escrito de Bálsamo estaba lleno de «ataques personalísimos» los cuales lo habían ofendido profundamente. Tras dudar de la «personalidad moral» del autor, lo invitó a participar de una polémica periodística seria, pues, según él, «por el camino de las chirigotas y las bufonadas no llegaríamos a parte alguna» ${ }^{70}$.

En un postrero intercambio de opiniones, Bálsamo volvió a hacer uso del humor. Tras mostrarse sorprendido ante la indignación de Melcior, rechazó participar de la polémica periodística porque tenía «la íntima convicción de que poniendo a contribución los nervios, los centros nerviosos, las células, los lóbulos y circunvoluciones cerebrales y el periespíritu, etc., etc., nos haríamos pesados a los lectores» ${ }^{71}$. Más allá de lo entretenidas que resulten estas cartas, la controversia que crearon evidencia el malestar que provocaban las nuevas teorías sobre la mediumnidad en algunos espiritistas, sobre todo las relacionadas con una noción del inconsciente aun ambigua.

\footnotetext{
66. Edelman, n. 54, p. 39-62.

67. Bálsamo, n. 64, p. 457-460.

68. Arregui, Jorge Vicente; Choza, Jacinto. Filosofía del hombre. Una antropología de la intimidad. Madrid: Rialp; 2002, p. 305-316.

69. Bálsamo, n. 64, p. 460.

70. Melcior, n. 62, p. 490-491.

71. Bálsamo, Dr. Carta del Dr. Bálsamo. Luz y Unión. 1901; 2 (54): 515.
} 


\section{Esa quisicosa llamada el inconsciente del médium}

La inquietud de Bálsamo respecto a las teorías del inconsciente es comprensible. Como puntualizó en su primera carta, si se aceptaba que servían para explicar los fenómenos espíritas: «habría que modificar radicalmente el concepto general e histórico del Espiritismo» ${ }^{72}$. Este razonamiento resulta muy acertado. Como se ha resaltado al principio, en el espiritismo el médium era considerado como un agente pasivo, un simple canal para la comunicación espiritual. Por ello, aceptar que el inconsciente del médium era el agente productor de los fenómenos espiritistas conllevaba modificar por completo la doctrina.

Por sí sola, esta afirmación hubiera servido para destapar la contradicción en la cual se caía si se intentaba defender esta concepción del médium desde el espiritismo. Sin embargo, en su carta Bálsamo hizo una aseveración acaso más reveladora. En sus palabras: «esta teoría [del inconsciente] que no vacilamos en calificar de errónea, resulta trascendentalmente funesta, porque viene patrocinada por los elementos intelectuales del Espiritismo» ${ }^{73}$. Se refería al hecho, claramente apuntado por Ellenberger, de que las distintas teorías del inconsciente se desarrollaron, en parte, mediante la observación de los fenómenos espíritas ${ }^{74}$. Por ejemplo, en el contexto inglés, la noción de Michael Faraday (1791-1867) de la «cerebración inconsciente» se planteó en relación a las mesas giratorias; asimismo, el concepto de «mente subliminal» de Frederic W. H. Myers se vio influenciado por el espiritismo anglosajón ${ }^{75}$. Por otro lado, en Francia las distintas nociones del inconsciente y la sugestión, como el subconsciente de Janet, estuvieron marcadas por la observación de fenómenos espiritistas como la escritura automática $^{76}$. El propio Janet admitió que, para estudiar el fenómeno de

\footnotetext{
72. Bálsamo, n. 64, p. 458.

73. Bálsamo, n. 64, p. 458

74. Ellenberger, n. 10.

75. Sobre las mesas giratorias y las teorías de Faraday véase: González de Pablo, Ángel. Sobre los inicios del espiritismo en España: La epidemia psíquica de las mesas giratorias de 1853 en la prensa médica. Asclepio. 2006; 58 (2): 63-69. Sobre Myers y la mente subliminal véase: Shamdasani, n. 11, p. 100-131.

76. Brower, Matthew Brady. Unruly spirits: The science of psychic phenomena in modern France. Urbana: University of Illinois Press; 2010.
} 
las múltiples personalidades, muchos habían sido los autores que habían empezado por asistir a sesiones espiritistas ${ }^{77}$.

Resulta interesante constatar cómo, para Bálsamo, la noción del inconsciente resultaba incompatible con la idea kardecista del periespíritu ${ }^{78}$. Sin embargo, para otros espiritistas o simpatizantes del movimiento, como Quintín López Gómez (1864-1934) y el propio Melcior, ambos conceptos eran complementarios ${ }^{79}$. Según ellos, el inconsciente estaba intrínsecamente ligado al periespíritu, ya que éste era el receptáculo de la conciencia, del cual el inconsciente formaba parte. En palabras de Melcior, el periespíritu se convertía en un «archivo de todos los actos y pensamientos que hemos realizado y tenido en la vida» ${ }^{80}$, los cuales acompañarían al hombre en sus varias reencarnaciones. Esta clase de asociación entre el periespíritu y el inconsciente sirvió para justificar la teoría de la supervivencia de la conciencia tras la muerte ${ }^{81}$.

Entonces, la noción del inconsciente no supuso per se una amenaza para el espiritismo, sino más bien el uso que se hizo de ella a través de teorías como el desdoblamiento de la personalidad o la fuerza psíquica, las cuales negaron la existencia de la mediumnidad espiritista. El problema de definir la «verdadera» mediumnidad, esto es, aquella en la cual intervenían los espíritus, se convirtió en una prioridad para el espiritismo. Gabriel Delanne así lo planteó en el Segundo Congreso Espiritista y Espiritualista celebrado en Paris en 1900, donde destacó la importancia de establecer la diferencia entre los hechos relacionados con el inconsciente, como la autosugestión, y los debidos a la intervención de los espíritus ${ }^{82}$. Eugenio García Gonzalo, médico y espiritista barcelonés, también insistió en diferenciar estos fenómenos. En su opinión, el hecho de que tantas autoridades científicas se hubieran interesado en el espiritismo había generado tanto esperanzas como frustraciones entre sus correligionarios. En un intento por calmar

\footnotetext{
77. Janet, n. 45, p. 126.

78. Para los espiritistas, el hombre era el resultado de una triple unión entre cuerpo, periespíritu y espíritu. El periespíritu era definido como un cuerpo fluídico que unía las dos partes y partía con el espíritu en el momento de la desencarnación. Véase, por ejemplo: Sharp, n. 24.

79. López Gómez, Quintín. Filosofia (3a ed). Barcelona: Maucci; s.d., 1919?, p. 155-199.

80. Melcior, Víctor. Los estados subconscientes y la aberraciones de la personalidad. Barcelona: Carbonell y Esteva; 1904, p. 120.

81. Sobre este tema véase la nota 24

82. Delanne, Gabriel. Congreso espiritista y espiritualista de 1900. Luz y Unión. 1900; 1 (9): 84-85 (85).
} 
los ánimos, afirmó que los científicos interesados en el tema habían de «inventar una y mil hipótesis» ${ }^{83}$ antes de admitir la comunicación espiritual.

\section{Conclusiones}

La definición de la mediumnidad fue uno de los aspectos fundamentales del espiritismo: de ella dependió la forma de entender su doctrina. Personalidades científicas reacias al espiritismo difundieron definiciones contrarias, generalmente basadas en un intento de patologizar y con ello, deslegitimar, la mediumnidad. Estos serían los casos, tan estudiados, de Pierre Janet o Gustave Le Bon ${ }^{84}$. Sin embargo, no debe incluirse a Víctor Melcior en este grupo. Como se ha comentado, aun sin ser un «espiritista ortodoxo» se sintió ligado a algunos aspectos de su doctrina, como la reencarnación. Asimismo, fue un miembro apreciado dentro del movimiento, participante activo en distintos grupos de investigaciones psíquicas y conferenciante invitado en muchos centros espiritistas catalanes. Como prueba de que Melcior mantuvo su interpretación sobre la mediumnidad tras la polémica con Bálsamo cabe mencionar las conferencias que dio, en 1905, en varios de estos centros. Las charlas estuvieron dedicadas al estudio del inconsciente, entendido como un estado particular de la conciencia. Entre otras cuestiones, reiteró que si el inconsciente no existiese, el espiritismo no tendría razón de ser, pues no se registrarían las experiencias vividas y una encarnación no tendría relación con la anterior. Asimismo, aseguró que solo mediante el inconsciente del médium y su fuerza psíquica podían explicarse los fenómenos atribuidos a los espíritus. Terminadas las conferencias, el presidente del centro Amor y Ciencia, Ángel Aguarod (1860-1932), dijo que no quería emitir ningún juicio al respecto. En mi opinión, su silencio expresa tanto su desacuerdo como su intención de no entrar en polémica con Melcior. Como en Bálsamo, la actitud de Aguarod debió estar motivada por la percepción de que las nuevas teorías médico-psicológicas representaban una

83. García Gonzalo, Eugenio. La psicofísica y el espiritismo. Revista de Estudios Psicológicos. 1897: 296-298 (297).

84. Lachapelle, Sofie. Investigating the supernatural: from spiritism and occultism to psychical research and metapsychics in France, 1853-1931. Baltimore: Johns Hopkins University Press; 2011. 
amenaza para la mediumnidad espiritista, en tanto se apropiaban del eje central de su doctrina ${ }^{85}$.

El caso de Melcior, expuesto a modo de microhistoria a través de los artículos sobre el fenómeno de la dinamita, nos muestra que el espiritismo no fue un movimiento uniforme. Desde partidarios de la noción del inconsciente y la fuerza psíquica en relación al espiritismo, a férreos detractores, tuvieron cabida en una corriente que destacó por su apertura de mente y tolerancia ${ }^{86}$. Sin embargo, algunas de las teorías que defendió Melcior, como la relación entre la mediumnidad y las psicopatías, no siempre fueron bien recibidas por los espiritistas. Por ello, no es de sorprender que en los años veinte del pasado siglo, con el auge de la metapsíquica en España ${ }^{87}$, Melcior terminara vinculado a este movimiento liderado por Richet, el cual se postuló como la alternativa francesa al «psychical research» anglosajón ${ }^{88}$. Como en el caso de Melcior, la aproximación de Richet a la mediumnidad se basó en la creencia de unas fuerzas o facultades inteligentes desconocidas en el hombre, capaces de producir fenómenos propios del espiritismo ${ }^{89}$. Sin tener en cuenta el componente psicopatológico, la mediumnidad entendida por Richet encaja con los prodigios del hombre encarnado defendidos por Melcior.

La redefinición de la mediumnidad -y sus fenómenos- mediante teorías como el desdoblamiento de la personalidad o la fuerza psíquica tuvo importantes consecuencias para el espiritismo. En algunos autores, como Melcior, se produjo un replanteamiento que no negó, de por sí, la hipótesis espírita. Sin embargo, en otros, como Richet o Janet, implicó su rechazo. De ahí que kardecistas convencidos, como Bálsamo, denunciaran que sus teorías no solo replanteaban los conceptos del espiritismo, sino que se los apropiaban para defender sus intereses particulares. En este sentido, la redefinición de la mediumnidad no fue un proceso neutro o consensuado. Sin embargo, la consecuencia fue unánime: el médium pasó a convertirse en un sujeto de investigación y su mediumnidad, entendida como productora de los fenómenos, devino el objeto científico a investigar.

\footnotetext{
85. Anónimo. Conferencias del Doctor Melcior. Lumen. 1905; 20: p. 84-89.

86. Horta, n. 3.

87. Mülberger y Balltondre, n. 3.

88. Marmin, Nicolas. Métapsychique et psychologie en France (1880-1940). Revue d'Histoire des Sciences Humaines. 2001; 1 (4): 145-171.

89. Richet, n. 45, p. 5 .
} 


\section{Agradecimientos}

Agradezco a Annette Mülberger y a Agustí Nieto-Galan sus comentarios a las versiones previas de este artículo. 
